\title{
Evaluation of Site-Diversified, Fully Functionalized Diazirine Probes for Chemical Proteomic Applications
}

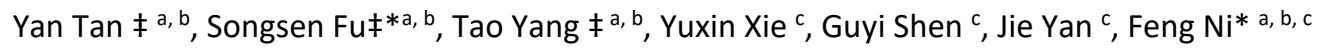 \\ a Institute of Drug Discovery Technology, Ningbo University, Ningbo 315211, China. \\ b Qian Xuesen Collaborative Research Center of Astrochemistry and Space Life Sciences, Ningbo University, Ningbo 315211, China. \\ ' LeadArt Technologies, Ningbo, 315211, China. \\ $\neq$ These authors contributed equally \\ *Corresponding author: S. F. Email: fusongsen@nbu.edu.cn; F. N. Email: nifeng@nbu.edu.cn;
}

\begin{abstract}
Photoaffinity probes combined with the chemical proteomic platform have emerged as versatile tools for ligand and target discovery. However, photoaffinity probes with retained activity cannot always label the known target, indicating that it is challenging to profile a ligand's targets based on its photoaffinity probe modified at a single site. Herein, we construct a series of site-diversified probes (P1-P6) of 4-anilinoquinazoline, a scaffold shared by several marketed EGFR-targeted drugs, via attaching a "fully functionalized" diazirine tag to six different sites, respectively. Chemical proteomic analysis revealed that these probes show different proteome-wide profiles and distinct competition patterns by erlotinib. Remarkably, low activity P4 towards EGFR inhibition has better EGFR labelling efficiency than the higher one, P5, which highlights the dominance of labelling accessibility of diazirine over probe affinity. In addition, the integrated analysis of protein targets of site-diversified probes can also help distinguish false positive targets. We anticipate that site-diversification of the probes of a given scaffold is an indispensable strategy to truly harness the power of photoaffinity-based chemoproteomics in drug discovery.
\end{abstract}

\section{Introduction}

Photoaffinity probes combined with the quantitative chemical proteomic platform have emerged as versatile tools for mapping reversible small molecule-protein interactions in living cells. Small molecules such as drugs, ${ }^{1-4}$ natural products, $^{5-9}$ synthetic hit compounds ${ }^{10-15}$ and fragments ${ }^{16-19}$ have been successfully converted into photoaffinity probes and subjected to target proteins mapping. These interaction data, on the one hand, provided valuable information for elucidating the mode of action as well as the adverse effects of bioactive small molecules, and on the other hand, greatly extended the members of "ligandable" proteins. Typically, a photoaffinity probe contains three parts: a scaffold that drivies the probe to approach targeted proteins, a photoreactive group that forms a covalent bond with amino acids upon radiation, and a reporter group (or "handle") for subsequent imaging or enrichment. For the reporter handle, traditional fluorophore or biotin are replaced with azide or alkyne, which could be readily post-derivatized via copper-catalyzed azide-alkyne cycloaddition (CUAAC) reactions; ${ }^{20}$ while the aliphatic diazirines are more and more widely utilized as photoreactive groups over aromatic diazirines and benzophenone during recent years. Furthermore, to retain most of the biological activity of parent molecule as well as biophysical properties (e. g. cell membrane penetrability), photoreactive group and reporter handle were evolved towards "fully functionalized" tags, represented by "minimalist" tags, ${ }^{21-24}$ wherein they are assembled within one tag which could be coupled to the scaffold in single synthetic step at one site. 
While applications of the "fully functionalized" diazirine tags are prevailing, there are several independent cases where these probes failed to label the known targets of their parent molecules regardless of the retained activities of probes and/or overexpression of the anticipated targets ${ }^{1,6,13}$. Indeed, compared to covalent probes, diazirinebased probes raised concerns about their labelling efficiency upon UV irradiation. It is well known that the solventquenching of carbene intermediate serves as a remarkable competitive side reaction. Recently, a comparative study of different "fully functionalized" diazirine tags found that tag has a substantial impact on labelling pattern of photoaffinity probes. ${ }^{25} \mathrm{~A}$ series of studies focused on the diazirine reactivity indicated significant amino acid labelling preferences of diazirine. ${ }^{26-29}$ Therefore, good binding between probe and target protein(s) cannot guarantee the accessibility of diazirine to protein residues, which might partially explain the false-negative labelling issue. The impact of tag position on probe's labelling efficiency could be much more prominent, however, researchers usually focus on the binding or activity retention of a candidate probe when apply it to proteome-wide profiling campaign. To the best of our knowledge, systematic investigation of the impact of tag positions on proteome-wide profiles of a given scaffold has not yet been reported. Herein, we constructed a panel of sitediversified probes based on a shared scaffold of several EGFR inhibitory drugs, and compared their performances on proteome-wide profiles, as well as EGFR enrichment and competitive displacement by erlotinib.

\section{Results and Discussion}

\section{Design and synthesis of site-diversified probes}

4-anilinoquinazoline is a scaffold shared by several $1^{\text {st }}$ and $2^{\text {nd }}$ generation EGFR-targeted drugs (Fig $\left.1 \mathrm{~A}\right)$. Given that these drugs have significant impacts on the current cancer treatment market, we thus chose 4-anilinoquinazoline as a scaffold to construct a set of multi-site probes P1- P6 by attaching the most frequently used "minimalist" tag ${ }^{21}$ at six different positions of this scaffold (Fig 1B), including two probes modified on sites $2^{\prime}$ and $8^{\prime}$ where no substitutions are introduced at original drugs. The six site-diversified probes, with identical charges and log $\mathrm{P}$ (except for P3), thus constituted a good probe toolkit for the evaluation of proteome-wide profiling and knowntarget labelling with potentially varied binding activities. Due to the lack of available handles on 4anilinoquinazoline for derivatization, we turned to the de-novo synthesis of six amine-substituted scaffolds (scheme S1-S5), which were subsequently conjugated with the carboxylic linker (L1) using amide linkage chemistry to give the corresponding probes P1-P6 (scheme S6). In addition, an aniline-derived compound, NP, was also synthesized, serving as the negative probe ${ }^{11,21}$ (scheme S7).

\section{Proteome-wide profiles of site-diversified probes P1-P6}

With these probes in hand, we first qualitatively assessed overall proteome profiles for each probe using gel-based imaging. In brief, Hela cells were incubated with individual probes $(2.5,5$, and $10 \mu \mathrm{M}, 30 \mathrm{~min})$ and exposed to UV light $(365 \mathrm{~nm}, 10 \mathrm{~min})$, then were harvested, lysed, and visualized by in-gel fluorescence following click chemistry with Azide-TAMRA (Fig 1C). The protein labelling of all probes presented a concentration-dependent manner, and 
A

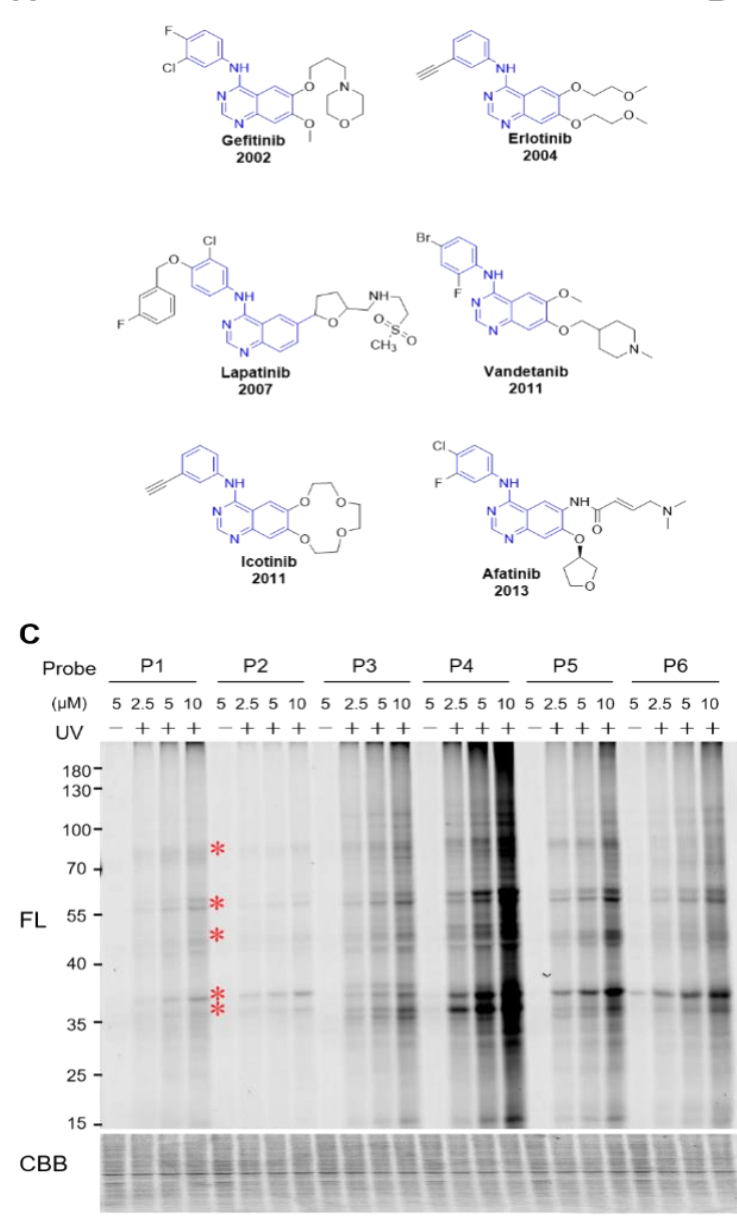

B

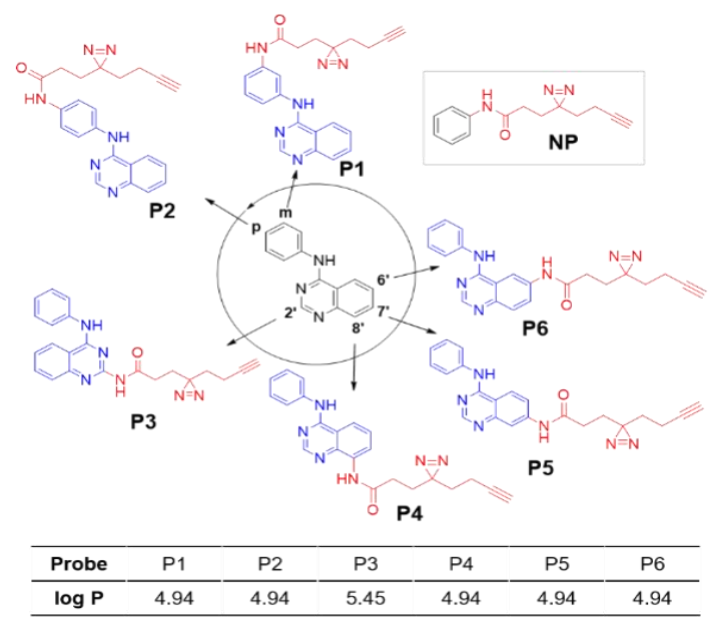

D

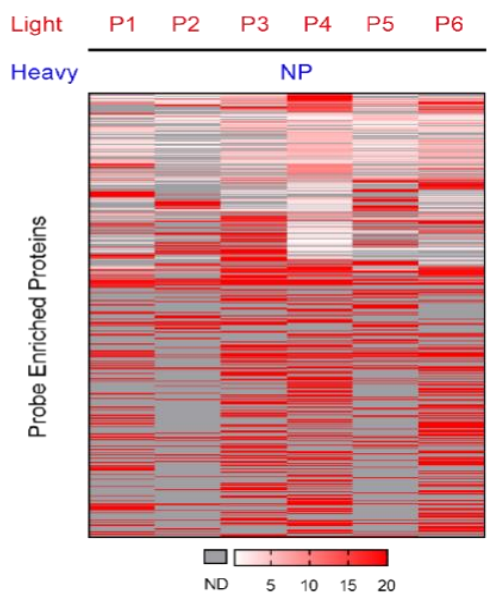

Fig 1.Design and proteome-wide profiles of site-diversified probes based on 4-anilinoquinazoline. (A) structures of some marketed EGFR inhibitors with a 4-anilinoquinazoline scaffold (blue). (B) Structures of site-diversified probes and negative probe (NP) were used in this study. Table shows the predicted logP of probes P1-P6. (C) Gel-based profiling of probe-protein interaction and probe show concentration-dependent increases in protein labelling in Hela cells. CBB-staining indicated the same amount of protein in each lane. (D) Heatmap display of SILAC ratios of relative protein enrichment of P1-P6 probe $(5 \mu \mathrm{M})$ versus NP $(5 \mu \mathrm{M})$ in Hela cells.

negligible labelling was observed in the absence of UV light. The labelling intensities and events varied among these probes, with P4 as the most prominent and P2 as the weakest ones. Except for some unique labelling events, we also observed several common labellings of strong intensity across six probes (Fig $1 \mathrm{C}$, asterisk).

Next, we set out to globally map each of the probe-protein interactions by MS-based quantitative chemical proteomics. We applied the stable isotope labelling of amino acids in cell culture (SILAC) approach and treated cells labeled with light or heavy isotopes with test probes (P1-P6) or NP (5 $\mu \mathrm{M}$ for each probe), respectively. Cells were lysed, equal protein amounts from NP and probe-treated samples pooled, and whole proteomes clicked to biotin azide. After enrichment and tryptic digest, the samples were analyzed by high-resolution liquid chromatography tandem mass spectrometry (LC-MS/MS). Each group was conducted in three stringent biological replicates (Fig. S1). Proteins that exhibited mean light : heavy SILAC ratios $\geq 5$ with a $p$-value of $<0.05$ were designated as preferred targets of the test probe. In aggregate, around 550 protein targets were identified for our whole site-diversified probe panel, which displayed a broad range of protein enrichments (Fig 1D, Fig S2, table S1). 


\section{Comparison of EGFR labelling by probes P1-P6}

We initially inspected the anticipated target, EGFR, and compared the relative abundance of enriched EGFR by our panel probes. It was observed that P6 and P4 perform the best, followed by P5, P3, and P1, while P2 gives a nondetectable signal (Fig 2A). To dissect the factors of probe affinity and photo-crosslinking efficiency, an in vitro EGFR kinase inhibitory activity, which could approximately reflect corresponding binding affinity, was measured for P1P6 and erlotinib. As expected, probes P5 and P6, modified at tunable sites in drug scaffold, retained modest inhibitory activities with an $\mathrm{IC}_{50}$ of $79.6 \mathrm{nM}$ and $9.6 \mathrm{nM}$, respectively. While $\mathrm{P} 3$ and P4 modified at unfavorable sites lost most of activities $\left(\mathrm{IC}_{50}>150 \mu \mathrm{M}\right)$; two other site-derived products, $\mathrm{P} 1$ and $\mathrm{P} 2$, are less active and almost inactive, respectively (Fig 2B, Fig S3). It is worth mentioning that P4 can still robustly enrich EGFR, though it is a much weaker EGFR inhibitor than P5 and P6. To construct the probable binding poses of P1-P6 in the EGFR complex structures, we conducted "blind" docking (without a designated pocket) using AutoDock Vina based on the crystal complex structure of EGFR and erlotinib. ${ }^{30}$ The results indicated that all six compounds chose to interact with the ATP binding pockets of EGFR. Considering that 4-anilinoquinazoline scaffold played a dominant role in competing with ATP, the complexes of EGFR and P1-P6 was stacked with erlotinib respectively (Fig S4), which show that the scaffold of P5 and P6 aligns nicely with erlotinib, while P1-P4 showed less favorable or poor overlapping with erlotinib. More specifically, 4-anilinoquinazoline of P4-P6 showed increasing overlap with that of erlotinib (Fig 2C), which is consistent with their increasing in vitro enzyme activity. We then took attention to the positions of their diazirine moiety. The diazirine of P5 extends to the edge of the pocket, while the diazirine of P4 and P6 were still wrapped in the pocket (Fig 2D). The diazirine of P4 was in a relatively negative environment, which may explain its

A

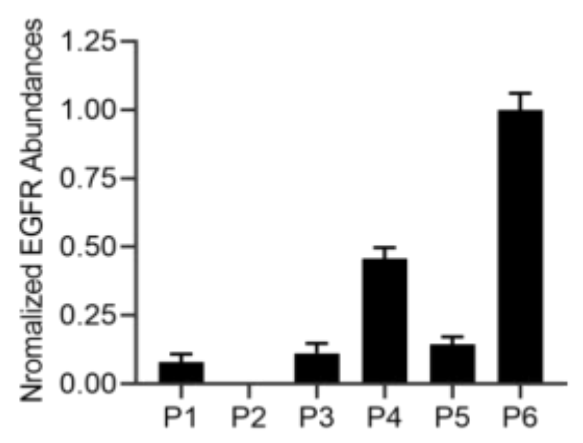

B

\begin{tabular}{|c|c|}
\hline Compound & EGFR: IC $_{50}$ \\
\hline $\mathrm{P} 1$ & $26.8 \mu \mathrm{M}$ \\
\hline $\mathrm{P} 2$ & $>150 \mu \mathrm{M}$ \\
\hline $\mathrm{P} 3$ & $>150 \mu \mathrm{M}$ \\
\hline $\mathrm{P} 4$ & $>150 \mu \mathrm{M}$ \\
\hline $\mathrm{P} 5$ & $79.62 \mathrm{nM}$ \\
\hline $\mathrm{P} 6$ & $9.635 \mathrm{nM}$ \\
\hline Erlotinib & $1.003 \mathrm{nM}$ \\
\hline
\end{tabular}

C

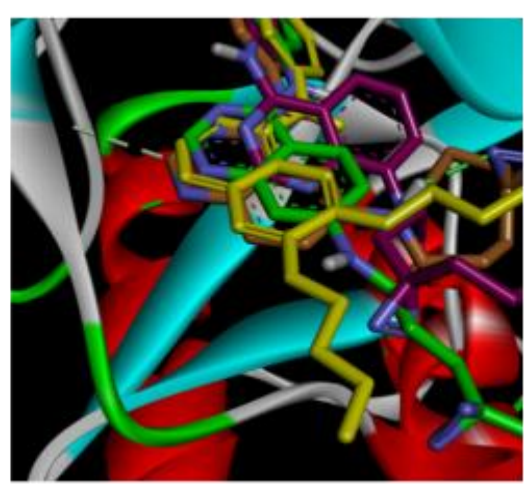

D

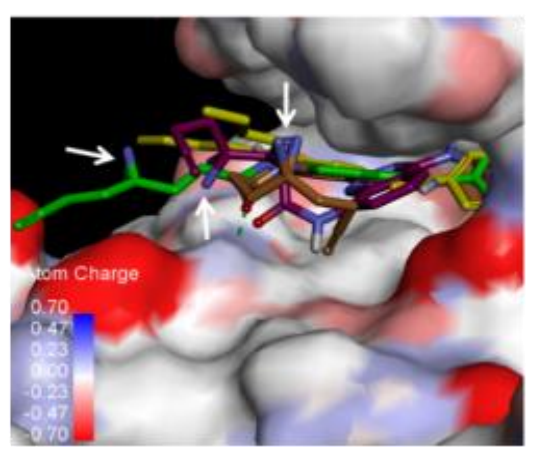

Fig 2. EGFR enrichment and inhibition by probes P1-P6. (A) Normalized mass abundances of EGFR enriched by probes P1-P6. Error bars represent standard deviations from three biological replicates. (B) IC $\mathrm{C}_{50}$ of P1-P6 and erlotinib against in vitro EGFR. (CD) Docking interaction of P4-P6 (P4: purple; P5: green; P6: brown) and erlotinib (yellow) with the active catalytic domain of EGFR. The position of diazirine of each probe was indicated by a white arrow. 
improved labelling efficiency based on a previous report that aliphatic diazirine preferentially labels acidic amino acids $^{26}$. Overall, our results micro-environmentally highlighted the importance of the photo-reactive accessibility of diazirine for target labelling or enrichment.

\section{Global analysis of protein targets of site-diversified probes P1-P6}

Next, we investigated the differences between proteome-wide profiles of our site-diversified probes. Remarkably, since the relatively small molecular weight of 4 -aniline quinazoline $(<300 \mathrm{Da})$, this set of site-diversified probes could actually serve as a small library of fully functionalized fragment (FFF) probes. In accordance with the in-gel results, P4 enriched the largest number of targets while P2 gave the minimal labellings (Fig 3A). In terms of target space, each probe has a unique group of enriched proteins (Fig 3A). Meanwhile, 12 proteins were found in the target lists of all probes (Fig 3A); Among all the pairwise comparisons, targets of P4 and P6 had the highest overlap ratio up to 33\% (Fig S5). In another aspect, proteins across a broad range of expression were enriched by P1-P6, of which P4 showed the best performance on enriching relative low abundance proteins (Fig S6). In terms of protein draggability, the majority of targets are not included in DrugBank (Fig 3B) and mainly cover enzymes, channels/transporters/receptors, transcription factors/regulators, and uncategorized proteins (Fig S7). In addition to EGFR, there were other six kinases that can be significantly enriched by our probe set (Fig S8). Taken together,

A

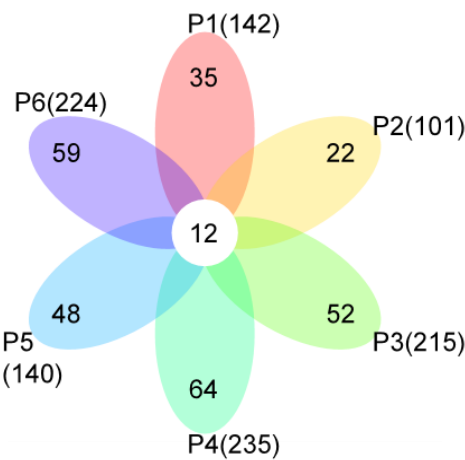

C

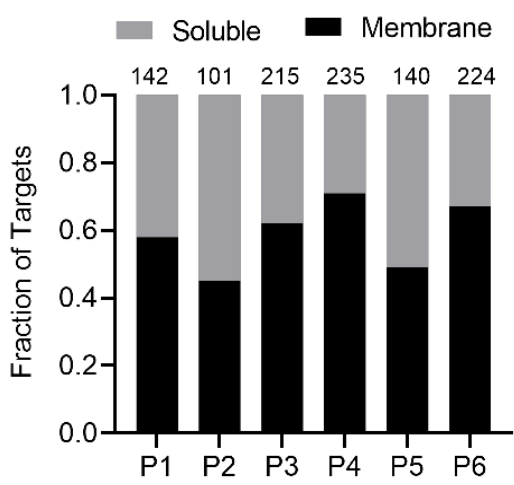

B

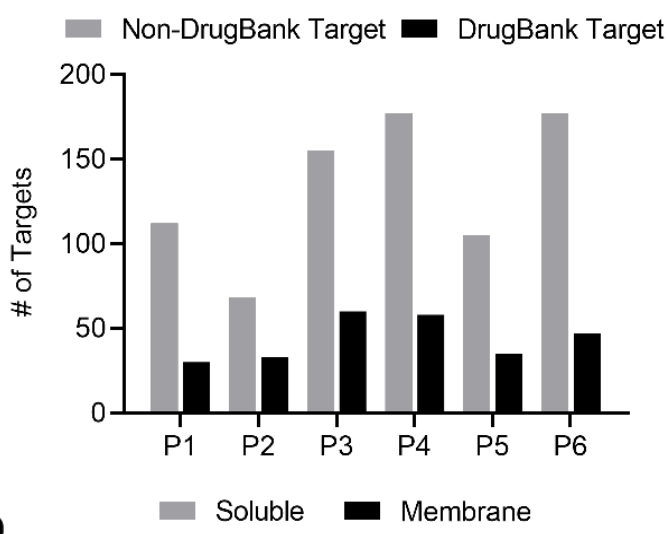

D

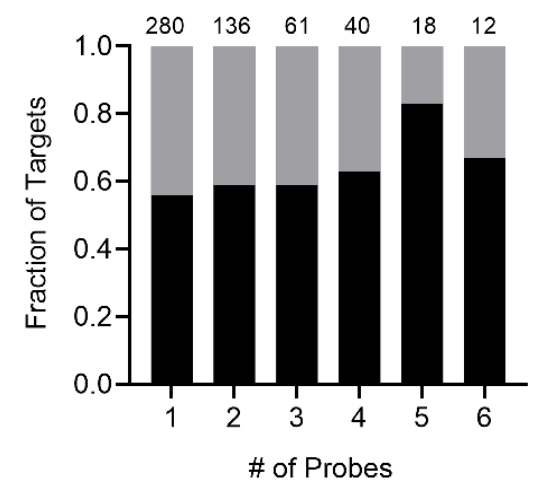

Fig 3. Global analysis of the targets of site-diversified probes. (A) Venn diagrams illustrating the protein hits of P1-P6 in Hela cells. (B) The probe enriched protein classification based on presence or absence in DrugBank. (C) The proportion of significantly enriched membrane and non-membrane proteins (membrane proteins are defined as those which possess one or more transmembrane domains as annotated by Uniprot) for each probe. (D) The number and ratio of membrane and non-membrane protein targets significantly enriched by different number of probes. 
Table 1. The protein targets enriched by at least 5 probes of P1-P6. The target genes in green font were potential targets of probes, while in red were well-known non-specific proteins.

\begin{tabular}{|c|c|c|c|c|c|c|c|c|c|c|}
\hline \multirow{2}{*}{ Gene } & \multicolumn{6}{|c|}{ SILAC Ratio (UV+/UV-) } & \multirow{2}{*}{ Protein Classification } & \multirow{2}{*}{ Membrane } & \multirow{2}{*}{ iBAQ } & \multirow{2}{*}{ \# of Probes } \\
\hline & P1 & P2 & P3 & P4 & P5 & P6 & & & & \\
\hline ATAD3B & 20 & - & 20 & 20 & 20 & 20 & Enzymes & TRUE & 5.38 & 5 \\
\hline ATP6AP2 & 20 & 20 & 20 & 20 & - & 20 & Channels, Transporters, Receptors & TRUE & 6.42 & 5 \\
\hline$A X L$ & 20 & 20 & 20 & 20 & 20 & - & Enzymes & TRUE & 4.62 & 5 \\
\hline CDK11A & 20 & 20 & 20 & 20 & 20 & 20 & Enzymes & FALSE & - & 6 \\
\hline CTSD & 15.47 & 19.60 & 20 & 20 & 20 & 17.92 & Enzymes & FALSE & 6.88 & 6 \\
\hline $\mathrm{ECH} 1$ & 2.65 & 9.68 & 7.19 & 20 & 7.28 & 8.44 & Enzymes & FALSE & 7.07 & 5 \\
\hline VDAC1 & 19.11 & 20 & 20 & 20 & 20 & 20 & Channels, Transporters, Receptors & TRUE & 8.11 & 6 \\
\hline VDAC2 & 9.13 & 8.32 & 20 & 20 & 20 & 19.23 & Channels, Transporters, Receptors & TRUE & 4.86 & 6 \\
\hline VIM & 8.23 & 6.57 & 5.85 & 9.19 & 5.69 & 6.70 & Uncategorized & FALSE & 8.74 & 6 \\
\hline COX7A2 & 20 & 20 & 20 & 20 & 20 & 20 & Enzymes & TRUE & 7.16 & 6 \\
\hline EPHX1 & 12.68 & 11.49 & 20 & 20 & 4.00 & 20 & Enzymes & TRUE & 7.00 & 5 \\
\hline GOLGB1 & - & 20 & 20 & 20 & 20 & 20 & Transcription factors, Regulators & TRUE & 6.03 & 5 \\
\hline LPCAT3 & 5.02 & 20 & 6.34 & 5.96 & 20 & - & Enzymes & TRUE & 6.66 & 5 \\
\hline MARCHF5 & 20 & 20 & 20 & 20 & 20 & 20 & Enzymes & TRUE & 6.51 & 6 \\
\hline $\mathrm{MTCH} 2$ & 6.02 & 3.25 & 5.31 & 7.20 & 8.71 & 9.50 & Channels, Transporters, Receptors & TRUE & 7.42 & 5 \\
\hline NIPA2 & - & 20 & 20 & 20 & 20 & 20 & Channels, Transporters, Receptors & TRUE & 5.26 & 5 \\
\hline NQO1 & 17.18 & 6.42 & 6.23 & 8.81 & 7.79 & 11.16 & Enzymes & FALSE & - & 6 \\
\hline OCIAD2 & 20 & - & 20 & 20 & 20 & 20 & Uncategorized & FALSE & 5.52 & 5 \\
\hline PEX16 & 20 & - & 20 & 20 & 20 & 20 & Uncategorized & TRUE & 5.68 & 5 \\
\hline PSAP & 7.95 & 5.25 & 17.68 & 15.02 & 12.32 & - & $\begin{array}{l}\text { Adapter, Scaffolding, Modulator } \\
\text { Proteins }\end{array}$ & FALSE & 7.06 & 5 \\
\hline SCARB1 & 20 & 20 & 20 & 20 & - & 13.43 & Channels, Transporters, Receptors & TRUE & 6.62 & 5 \\
\hline SLC16A3 & 6.82 & 8.87 & 6.24 & 10.71 & 3.54 & 8.95 & Channels, Transporters, Receptors & TRUE & 7.21 & 5 \\
\hline SLC18B1 & 20 & 20 & 20 & 20 & 20 & 20 & Channels, Transporters, Receptors & TRUE & - & 6 \\
\hline SLC25A10 & 11.68 & 11.07 & 14.09 & - & 16.09 & 18.15 & Channels, Transporters, Receptors & TRUE & 6.08 & 5 \\
\hline SLC25A15 & 20 & 20 & - & 20 & 20 & 20 & Channels, Transporters, Receptors & TRUE & 5.90 & 5 \\
\hline SLC25A20 & 16.96 & 16.96 & 20 & 19.63 & 19.62 & 20 & Channels, Transporters, Receptors & TRUE & 5.60 & 6 \\
\hline SPNS1 & 20 & 16.43 & 17.36 & 17.88 & 6.24 & 20 & Channels, Transporters, Receptors & TRUE & 4.98 & 6 \\
\hline SSR1 & 9.59 & 5.32 & 7.97 & 19.56 & 13.11 & 18.06 & Uncategorized & TRUE & 7.11 & 6 \\
\hline TMEM259 & 20 & - & 20 & 20 & 20 & 20 & Uncategorized & TRUE & 5.69 & 5 \\
\hline TOMM22 & 17.97 & - & 20 & 20 & 20 & 20 & Channels, Transporters, Receptors & TRUE & 7.66 & 5 \\
\hline
\end{tabular}

through the comprehensive utilization of site-diversified probes, we can significantly improve the analysis depth of protein interaction profiles of a given drug scaffold.

In terms of subcellular localization, targets of P1-P6 presented different patterns. P4 and P6 showed preferential enrichment for membrane proteins (Fig 3C). Interestingly, among the proteins commonly enriched by 5 or 6 probes, the proportion of membrane proteins was high. Considering the shape diversity of site-diversified probes and the recently reported preferential enrichment of membrane proteins by aliphatic diazirine, these labelling events may be regarded as low scaffold-dependent. In fact, several well-known background proteins enriched by photoaffinity tags $^{31-33}$ were in the list of table 1, including VDAC1, VDAC2, CTSD, ECH1, and VIM. In addition, a number of solute carrier proteins $(S L C)$ were also identified in the list, likely attributed to the broad substrate scope of this protein family members and implies the potential challenge to develop their specific probes ${ }^{34,35}$. The highly efficient labelling of SLCs by our probes could probably be explained by the "probe passing and fixed" mechanism. We believe that this protein list is a useful resource for suspicious targets in other target identification projects.

\section{Competitive displacement of site-diversified probes by erlotinib}

Next, we utilized site-diversified probes to identify potential off-targets of the $1^{\text {st }}$ generation EGFR drug, erlotinib, through a competitive displacement assay. First, the in-gel approach was employed to qualitatively evaluate the effect of competition. We found that P4 showed a most significant wipe-off of fluorescent signal upon competition, followed by P3, and the others (Fig 4A). Genfitinib and vandetanib, were also tested and gave similar results (FigS9). Next, we used quantitative mass spectrometry to identify potential off-targets of erlotinib in living Hela cells. Briefly, 

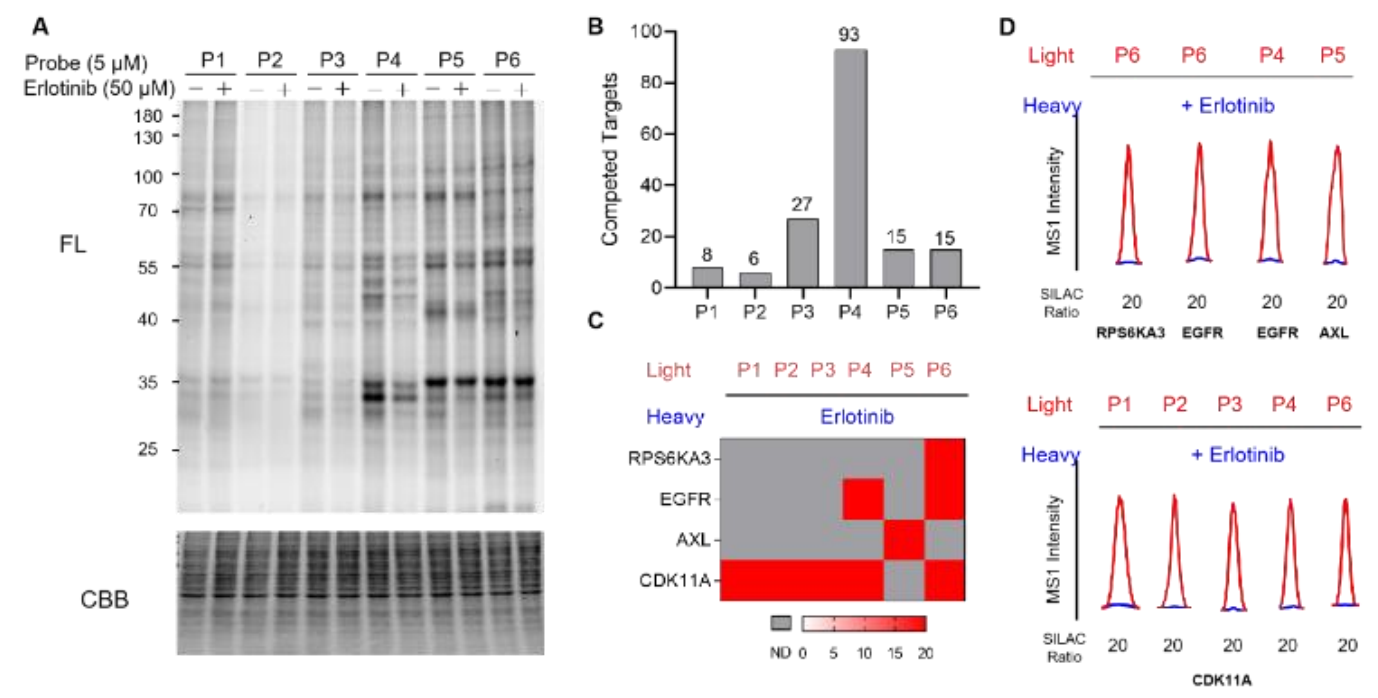

Fig 4. Competitive displacement of site-diversified probes with erlotinib as a competitor. (A) Gel-based competitive profiling of probes with 10x erlotinib $(50 \mu \mathrm{M})$ as a competitor. (B) The number of potential targets of erlotinib identified by each probe. (C) Heatmap showing fully engagement of several kinases by erlotinib in living Hela cells. (D) Representative MS1 peptide traces for kinase targets of erlotinib in competition experiments.

we treated isotopically light and heavy cells with probe $(5 \mu \mathrm{M})$ and either DMSO or $10 \times$ erlotinib $(50 \mu \mathrm{M})$ respectively, followed by radiation, lysis, click with biotin-N3, enrichment, and LC-MS/MS analysis (Fig S10). Proteins that showed mean light : heavy ratios of $\geq 2.0$ with a $p$-value of $<0.05$ were considered substantially engaged by erlotinib (Fig S11, table S2). Most off-target proteins were identified in P4 group (Fig 4B), which was in line with the gel results. In addition to EGFR, three other kinases (RPS6KA3, AXL, and CDK11A) identified were fully engaged $(L / H \geq 20)$ by erlotinib at the indicated concentration $(50 \mu \mathrm{M})$ (Fig 4C, 4D). With the list of potential offtargets of erlotinib, we interrogated those proteins that existed in table 1. Except for kinases (AXL and CDK11A) and ATPase related proteins (ATAD3B and ATP6AP2), which were fully engaged with erlotinib (L/H $\geq 20)$, most of other proteins were not highly occupied by erlotinib (table S2). Interestingly, typical false positive proteins such as VDAC1, VDAC2 and CTSD gave very low competitive ratios, which suggested that a combination of quantitative competition data of site-diversified probes could help to confirm false-positive targets of a given competitor.

\section{Discussion}

In principle, chemical proteomic is a tool to quantitatively sequence the surrounding proteins of a give chemical moiety. Maximizing the proteomic MS signal, a powerful probe that can efficiently enrich the target proteins is vital, and it may require systematic optimization of tagging site, linkage, and photoreactive group. To focus on the impact of tagging site on target enrichment, we chose the drug scaffold, 4-anilinoquinazoline, and designed a set of 6-member site-diversified probe library. We disclosed that these site-diversified probes with the same charges and almost the same lipophilicity gave both characteristic and overlapped proteome interaction profiles in living cells. The combination of individual data makes the interaction map of the scaffold more complete for subsequent target identification. For fragment probes, one major goal is to collect as many landscapes as possible to expand the scope of "ligandable" proteins ${ }^{17,}{ }^{18}$. Due to the relatively low affinity of fragments and the uncertainty of labelling efficiency, obviously, fragment probe based on a single site tagging have limitations to explore its whole 
target space, while site-diversified fragment probes can draw a larger target space, thus providing more fragmentprotein binding data for fragment-based ligand discovery (FBLD). On the other hand, when utilized as affinity-based probes (AfBP) in competitive mode, site-diversified probes can provide greater coverage of potential off-targets, thereby providing more complete information for the interpretation of MOA of competitor drugs, as exemplified by erlotinib in this work. The common targets of site-diversified probes also call our attention to proteins such as background proteins (e. g. VDAC1, VDAC2, VIM), ${ }^{31,33}$ potential target proteins (e. g. SLC25A20), ${ }^{1}$ and ambiguous proteins (e. g. CTSD). ${ }^{31,36,37}$ Therefore, we suggest inspecting those proteins that commonly enriched by multiple probes in other target identification campaigns. The mechanism of multiple-ligand recognition of common proteins is still unclear. In the future, approaches such as hotspot identification may be useful to address the problem.

Currently, the prevailing principle of probe design is activity-oriented, but there is no consensus on the criteria. Recent studies suggested practical instructions on tag design, such as utilizing different types of tags ${ }^{25}$ and changing the electrostatic charge of the probe ${ }^{26}$. However, in our case of EGFR, it was found that the labelling efficiency of P5 is independent of its activity. Therefore, the selection of modification sites should be prior to the choice of tag. The power of photo-induced labelling enables the proteome-wide profiles of the fragment with moderate affinity $(>100 \mu \mathrm{M}),{ }^{18}$ thus there is sufficient flexibility to achieve activity-retention of any candidate probes. Especially when designing a tool AfBP for a desired protein, ${ }^{38,39}$ one may consider placing the tag in a position with certain steric hindrance to improve labelling accessibility, instead of completely exposing it to the solvent for retaining the maximal affinity of its scaffold.

Lastly, we also believe that reducing the distance between diazirine and the scaffold may be an alternative strategy to improve labelling efficiency, thus tags smaller than the current "minimalist" version are still worthy of developing. Taking advantage of the "minimalist" tags, site-diversified probe strategy will become a more attractive and practical option when the late-stage derivatization chemistry explodes. ${ }^{40}$

\section{Conclusions}

In summary, we have revealed that positions of diazirine tagging site on a scaffold have significant impacts on the protein profile of the resulting probes. Importantly, compared with the binding strength between probe and target, the labelling efficiency of the target protein is more significantly affected by the overall surroundings of the diazirine group. The site-diversified probe strategy provides an opportunity to achieve specific labelling of desired proteins by combinational position tuning and types of derivatized tag, representing an attractive direction in AfBP design. In addition, the integrated analysis of protein targets of those site-diversified probes not only unveils more complete target space but also helps distinguish false positive targets. Site-diversified probe strategy is a recommended solution for less biased target profiling and identification, especially for fragment molecules and target-unknown hits.

\section{Author Contributions}


S. F. and F. N. conceived of the research, analysed data and wrote the manuscript. Y. T., S. F., T. Y., Y. X., and J. Y. performed experiments, analysed data, and contributed to editing. G. S assisted with molecular docking analysis.

\section{Conflicts of interest}

J. Y. and F. N. are shareholders of LeadArt Technologies.

\section{Acknowledgements}

We gratefully acknowledge supports from National Natural Science Foundation of China (91856126, 21778042), Scientific Research Grant of Ningbo University (215-432000282) and Ningbo Top Talent Project (215-432094250).

\section{Notes and references}

1. C. G. Parker, C. A. Kuttruff, A. Galmozzi, L. Jorgensen, C. H. Yeh, D. J. Hermanson, Y. Wang, M. Artola, S. J. McKerrall, C. M. Josyln, B. Norremark, G. Dunstl, J. Felding, E. Saez, P. S. Baran and B. F. Cravatt, ACS Cent. Sci., 2017, 3, 1276-1285.

2. J. Gao, A. Mfuh, Y. Amako and C. M. Woo, J. Am. Chem. Soc., 2018, 140, 4259-4268.

3. Y. Chang, D. Zhu, H. Guo, X. Yin, K. Ding and Z. Li, ChemBioChem, 2019, 20, 1783-1788.

4. I. V. L. Wilkinson, K. J. Perkins, H. Dugdale, L. Moir, A. Vuorinen, M. Chatzopoulou, S. E. Squire, S. Monecke, A. Lomow, M. Geese, P. D. Charles, P. Burch, J. M. Tinsley, G. M. Wynne, S. G. Davies, F. X. Wilson, F. Rastinejad, S. Mohammed, K. E. Davies and A. J. Russell, Angew. Chem. Int. Ed., 2020, 59, 2420-2428.

5. J. J. Hulce, A. B. Cognetta, M. J. Niphakis, S. E. Tully and B. F. Cravatt, Nat. Methods, 2013, 10, 259-264.

6. S. Zhuang, Q. Li, L. Cai, C. Wang and X. Lei, ACS Cent. Sci., 2017, 3, 501-509.

7. C. E. Keohane, A. D. Steele, C. Fetzer, J. Khowsathit, D. Van Tyne, L. Moynie, M. S. Gilmore, J. Karanicolas, S. A. Sieber and W. M. Wuest, J. Am. Chem. Soc., 2018, 140, 1774-1782.

8. P. Le, M. B. Nodwell, J. Eirich and S. A. Sieber, Chem. Eur. J., 2019, 25, 12644-12651.

9. H. W. Kavunja, K. J. Biegas, N. Banahene, J. A. Stewart, B. F. Piligian, J. M. Groenevelt, C. E. Sein, Y. S. Morita, M. Niederweis, M. S. Siegrist and B. M. Swarts, J. Am. Chem. Soc., 2020, 142, 7725-7731.

10. B. Lim, J. Lee, B. Kim, R. Lee, J. Park, D. C. Oh, J. Gam and J. Lee, Asian J. Org. Chem., 2019, 8, 1626-1630.

11. N. Ma, Z. M. Zhang, J. S. Lee, K. Cheng, L. Lin, D. M. Zhang, P. Hao, K. Ding, W. C. Ye and Z. Li, ACS Chem. Biol., 2019, 14, 2546-2552.

12. I. A. Stepek, T. Cao, A. Koetemann, S. Shimura, B. Wollscheid and J. W. Bode, ACS Chem. Biol., 2019, 14, 1030-1040.

13. R. T. Howard, P. Hemsley, P. Petteruti, C. N. Saunders, J. A. Molina Bermejo, J. S. Scott, J. W. Johannes and E. W. Tate, ACS Chem. Biol., 2020, 15, 325-333.

14. C. L. Gao, G. G. Hou, J. Liu, T. Ru, Y. Z. Xu, S. Y. Zhao, H. Ye, L. Y. Zhang, K. X. Chen, Y. W. Guo, T. Pang and X. W. Li, Angew. Chem. Int. Ed., 2020, 59, 2429-2439.

15. C. R. Kennedy, A. Goya Grocin, T. Kovacic, R. Singh, J. A. Ward, A. R. Shenoy and E. W. Tate, ACS Chem. Biol., 2021, 16, 982-990.

16. J. S. Cisar and B. F. Cravatt, J. Am. Chem. Soc., 2012, 134, 10385-10388.

17. T. Kambe, B. E. Correia, M. J. Niphakis and B. F. Cravatt, J. Am. Chem. Soc., 2014, 136, 10777-10782. 
18. C. G. Parker, A. Galmozzi, Y. Wang, B. E. Correia, K. Sasaki, C. M. Joslyn, A. S. Kim, C. L. Cavallaro, R. M. Lawrence, S. R. Johnson, I. Narvaiza, E. Saez and B. F. Cravatt, Cell, 2017, 168, 527-541.e529.

19. Y. Wang, M. M. Dix, G. Bianco, J. R. Remsberg, H. Y. Lee, M. Kalocsay, S. P. Gygi, S. Forli, G. Vite, R. M. Lawrence, C. G. Parker and B. F. Cravatt, Nat. Chem., 2019, 11, 1113-1123.

20. V. V. Rostovtsev, L. G. Green, V. V. Fokin and K. B. Sharpless, Angew. Chem. Int. Ed., 2002, 41, 2596-2599.

21. Z. Li, P. Hao, L. Li, C. Y. J. Tan, X. Cheng, G. Y. J. Chen, S. K. Sze, H.-M. Shen and S. Q. Yao, Angew. Chem. Int. Ed., 2013, 52, 8551-8556.

22. Z. Li, D. Wang, L. Li, S. Pan, Z. Na, C. Y. Tan and S. Q. Yao, J. Am. Chem. Soc., 2014, 136, 9990-9998.

23. C.-F. Chang, A. Mfuh, J. Gao, H.-Y. Wu and C. M. Woo, Tetrahedron, 2018, 74, 3273-3277.

24. W. Lang, C. Yuan, B. Zhu, S. Pan, J. Liu, J. Luo, S. Nie, Q. Zhu, J. S. Lee and J. Ge, Org. Biomol. Chem., 2019, 17, 3010-3017.

25. L. P. Conway, A. M. Jadhav, R. A. Homan, W. Li, J. S. Rubiano, R. Hawkins, R. M. Lawrence and C. G. Parker, Chem. Sci., 2021, 12, 7839-7847.

26. A. V. West, G. Muncipinto, H. Y. Wu, A. C. Huang, M. T. Labenski, L. H. Jones and C. M. Woo, J. Am. Chem. Soc., 2021, 143, 6691-6700.

27. D. S. Ziemianowicz, R. Bomgarden, C. Etienne and D. C. Schriemer, J. Am. Soc. Mass Spectrom., 2017, 28, 2011-2021.

28. C. Iacobucci, M. Gotze, C. Piotrowski, C. Arlt, A. Rehkamp, C. Ihling, C. Hage and A. Sinz, Anal. Chem., 2018, 90, 2805-2809.

29. J. G. K. O'Brien, A. Jemas, P. N. Asare-Okai, C. W. Am Ende and J. M. Fox, Org. Lett., 2020, 22, 9415-9420.

30. O. Trott and A. J. Olson, J. Comput. Chem., 2010, 31, 455-461.

31. P. Kleiner, W. Heydenreuter, M. Stahl, V. S. Korotkov and S. A. Sieber, Angew. Chem. Int. Ed., 2016, 56, 13961401.

32. H. Park, J. Y. Koo, Y. V. Srikanth, S. Oh, J. Lee, J. Park and S. B. Park, Chem. Commun., 2016, 52, 5828-5831.

33. J. Park, M. Koh, J. Y. Koo, S. Lee and S. B. Park, ACS Chem. Biol., 2016, 11, 44-52.

34. A. Cesar-Razquin, B. Snijder, T. Frappier-Brinton, R. Isserlin, G. Gyimesi, X. Bai, R. A. Reithmeier, D. Hepworth, M. A. Hediger, A. M. Edwards and G. Superti-Furga, Cell, 2015, 162, 478-487.

35. W. W. Wang, L. Gallo, A. Jadhav, R. Hawkins and C. G. Parker, J. Med. Chem., 2020, 63, 3834-3867.

36. A. M. Zuhl, C. E. Nolan, M. A. Brodney, S. Niessen, K. Atchison, C. Houle, D. A. Karanian, C. Ambroise, J. W. Brulet, E. M. Beck, S. D. Doran, B. T. O'Neill, C. W. am Ende, C. Chang, K. F. Geoghegan, G. M. West, J. C. Judkins, X. Hou, D. R. Riddell and D. S. Johnson, Nat. Commun., 2016, 7, 13042.

37. J. Xu, Z. Zhang, L. Lin, H. Sun, L. V. White, K. Ding and Z. Li, ACS Med. Chem. Lett., 2020, 11, 535-540.

38. E. K. Grant, D. J. Fallon, H. C. Eberl, K. G. M. Fantom, F. Zappacosta, C. Messenger, N. C. O. Tomkinson and J. T. Bush, Angew. Chem. Int. Ed., 2019, 58, 17322-17327.

39. E. K. Grant, D. J. Fallon, M. M. Hann, K. G. M. Fantom, C. Quinn, F. Zappacosta, R. S. Annan, C. W. Chung, P. Bamborough, D. P. Dixon, P. Stacey, D. House, V. K. Patel, N. C. O. Tomkinson and J. T. Bush, Angew. Chem. Int. Ed., 2020, 59, 21096-21105.

40. Y. J. Lim, Y. Kuang, J. Wu and S. Q. Yao, Chem. Eur. J., 2021, 27, 3575-3580. 